\title{
ESTUDO DAS CARACTERÍSTICAS ESTRUTURAIS E TERMOFÍSICAS NAS ETAPAS DE FORJAMENTO ROTATIVO A QUENTE E A FRIO EM UMA LIGA DE NiTi*
}

\author{
Patrícia Freitas Rodrigues ${ }^{1}$ \\ Francisco Manuel Braz Fernandes ${ }^{2}$ \\ Andersan dos Santos Paula ${ }^{3}$ \\ Shimeni Baptista Ribeiro 4 \\ Edir Teixeira Neves ${ }^{5}$ \\ Saulo Brinco Diniz ${ }^{6}$
}

\section{Resumo}

Foram utilizadas neste estudo amostras de uma liga de $\mathrm{NiTi}$ rica em $\mathrm{Ni}$, a fim de observar as mudanças estruturais e termofísicas ao longo das etapas de forjamento rotativo, nas condições: Bruta de Fusão, Refundida e extraídas após distintas etapas do forjamento inicialmente (primeira, terceira) e ao fim a frio (quinta e sexta). As variáveis dos processamentos termomecânicos e a composição química são fatores de grande importância no processamento destas ligas, afetando a estrutura e por consequência nas transformações de fase, no efeito de memória de forma e na superelasticidade. Dessa forma, foram realizados ensaios de calorimetria diferencial de varredura (DSC), para definir as temperaturas de transformação de fases, e de difração de raio $X(D R X)$ para observar as fases presentes a temperatura ambiente.

Palavras-chave: Ligas NiTi; Superelasticidade; Transformação de Fase; Forjamento Rotativo.

\section{STUDY OF THE STRUCTURAL AND THERMOPHYSIC CHARACTERISTICS ON THE HOT AND COLD ROTARY FORGING STEPS IN A NI-RICH NITI ALLOY}

\section{Abstract}

Samples of NiTi-Ni alloys were used in this study to observe structural and thermophysical changes in rotary forging steps of the following conditions: Cast, Remelted and after different steps of the forging (first, third) and finally the cold (fifth and sixth). The variables of thermomechanical processing and the chemical composition are very important factors in the processing of these alloys, affecting the structure and consequently the phase transformations in shape memory effect and superelasticity. Thus, differential scanning calorimetric (DSC) test, to define the phase transformation temperatures, and X-ray diffraction (XRD) to observe the phases present at room temperature were done.

Keywords: NiTi Alloys; Superelasticity; Phase Transformation; Rotary Forging.

1 Bacharel em Química Industrial, M.Sc., Doutoranda em Ciência e Engenharia de Materiais, Cenimat/i3N, DCM/FCT/UNL, Caparica, Portugal; e Bolsista Capes; Bolsista, CAPES - Foundation Ministry of Education of Brazil, Brasília - DF - Brazil

2 Engenheiro Mecânico, M.Sc., D. Sc., Professor Associado, Cenimat/i3N, DCM/FCT/UNL, Caparica, Portugal.

3 Engenheira Metalurgista, M.Sc., D.Sc., Professora Adjunta, IME, Rio de Janeiro, RJ, Brasil; e orientadora credenciado do PPGEM/UFF, Volta Redonda, RJ, Brasi.

4 Engenheira Metalurgista, M.Sc., D.Sc., Professora, UniFOA, Volta Redonda, RJ, Brasil.

5 Engenheiro Metalurgista, M.Sc., Professor, UniFOA, Volta Redonda, RJ, Brasil

6 Engenheiro Metalurgista, M.Sc., Doutorando em Ciências dos Materiais, IME, Rio de Janeiro, RJ; Professor DI - 1, CEFET - Angra dos Reis, Angra dos Reis, RJ, Brasil. 


\section{INTRODUÇÃO}

As ligas com memória de forma que apresentam propriedades funcionais de elevado interesse, tais como: a superelasticidade e efeito de memória de forma, são classificadas como materiais avançados passíveis de transformação martensítica que adquirem a capacidade de retomar a uma forma ou dimensão previamente definida quando são submetidas a tratamentos termomecânicos apropriados.

Materiais com estas características podem ser deformados a uma temperatura baixa, em seu campo de estabilidade da fase martensítica e, após elevação de temperatura, até seu campo de estabilidade da fase austenítica, retornará à forma que apresentava, classificando assim, o efeito de memória de forma. Esses materiais quando são deformados no campo austenítico, se torna possível promover uma transformação de fase reversível do tipo martensítica induzida por tensão. Neste último caso, pode-se reverter a transformação quando se remove completamente a tensão aplicada, classifica-se assim a superelasticidade ${ }^{(1)}$.

Dentre estes materiais faz-se presente as ligas $\mathrm{NiTi}$, as quais apresentam alta resistência mecânica, à fadiga e à corrosão, boa ductilidade e biocompatibilidade, isso se for comparada com outras ligas com memória de forma ${ }^{(2)}$.

As temperaturas de transformação de fase em ligas NiTi ricas em Ni são observadas abaixo da temperatura ambiente, sendo geralmente superelásticas a temperatura ambiente ${ }^{(3)}$. Essas ligas apresentam a formação de até três tipos de fases, austenita (B2 - mais alta temperatura - cúbica do tipo $\mathrm{CsCl}$ ), fase $\mathrm{R}$ (martensita - temperatura intermediárias - trigonal com distorção romboédrica no ângulo a) e martensita (B19' - mais baixa temperatura - monoclínica)

As características do material são dependentes dos tratamentos termomecânicos que forma aplicados durante sua fabricação. Esses podem promover precipitação, redução de defeitos, ainda transformação de fase, relevantes às propriedades estruturais e funcionais ${ }^{(4)}$.

O maior desafio na produção dessas ligas se encontra no controle composicional, principalmente na redução de presença de impurezas, tais como carbono e oxigênio. Estas quando presentes na composição irão se combinar com o $\mathrm{Ni}$ e Ti, formando $\mathrm{TiC}$ e $\mathrm{Ti}_{4} \mathrm{Ni}_{2} \mathrm{O}$ (na fusão) e/ou $\mathrm{TiO}_{2}$ (por oxidação no estado sólido), decrescendo o teor efetivo de Ti e/ou Ni livre. Influenciando assim na formação das fases austenita e martensita, estas associadas às temperaturas de transformação de fase.

O processo de fusão aplicado neste estudo é o VIM (fusão em forno de indução à vácuo), este promove a fusão do material pelo aquecimento por indução de alta frequência em vácuo ou gás inerte. Com este processo é possível controlar a composição química ${ }^{(2,4)}$. Logo esta etapa é fundamental, uma vez que o controle de teor de níquel e de titânio pode ser controlado e os níveis de $\mathrm{O}$ e $\mathrm{C}$ minimizados.

O trabalho a quente, tal como o forjamento rotativo utilizado neste trabalho, é fundamental para obtenção de uma microestrutura adequada, com grande influência às propriedades, que serão refinadas no trabalho a frio e tratamentos térmicos posteriores.

O trabalho a quente é bem menos complexo que o trabalho a frio. A conformação está fortemente relacionada a composição química, quanto maior o teor de níquel mais resistente será à conformação. Ao apresentar teores superior a $51 \%$ at, o trabalho a frio será realizado de maneira complexa, pois apresentará um forte encruamento. Buscando minimizar o impacto do encruamento, se aplica tratamentos térmicos capazes de minimizar este efeito entre as etapas de trabalho a frio. 
O presente trabalho visou compreender as características termofísicas (temperaturas de transformação de fases) e as características estruturais à temperatura ambiente (fases presentes) após cada etapa de um processamento termomecânico de forjamento rotativo a quente e a frio, em escala piloto, de uma liga NiTi rica em Ni. As análises de Calorimetria Diferencial de Varredura (DSC) e difração de raio-X (DRX), foram utilizadas neste estudo com foco nas amostras extraídas a partira das condições: Bruta de Fusão, Refundida, Primeiro Forjamento Rotativo a Quente, Terceiro Forjamento Rotativo a Quente, Quinto Forjamento (quatro forjamentos a quente seguido de um forjamento a frio) e Sexto Forjamento (quatro a quente e dois forjamentos a frio).

\section{MATERIAL E MÉTODOS}

\subsection{Material}

O material em estudo é proveniente de uma liga $\mathrm{NiTi}$ rica em $\mathrm{Ni}(51,03 \%$ at $\mathrm{Ni})$, elaborado pelo professor e pesquisador Dr. Jorge Otubo do Instituto Tecnológica da Aeronáutica (ITA), Brasil. Este material foi submetido a uma rota de processamento termomecânico via forjamento rotativo (inicialmente a quente e posteriormente a frio), permitindo que fossem coletadas amostras para o presente estudo.

Um esquema para ilustrar o processamento é representado na Figura 1. As etapas do processo foram intercaladas por tratamentos térmicos específicos $\left(800^{\circ} \mathrm{C} / 10 \mathrm{~min}\right.$, simples reaquecimento prévio ao forjamento a quente e solubilização com resfriamento em água prévio ao forjamento a frio), com auxílio de duas forjas de forjamento rotativo: 4 martelos (para as duas primeiras e as grande reduções a quente) e 2 martelos (para as seguintes reduções, duas a quente, e as duas últimas e pequenas reduções a frio).

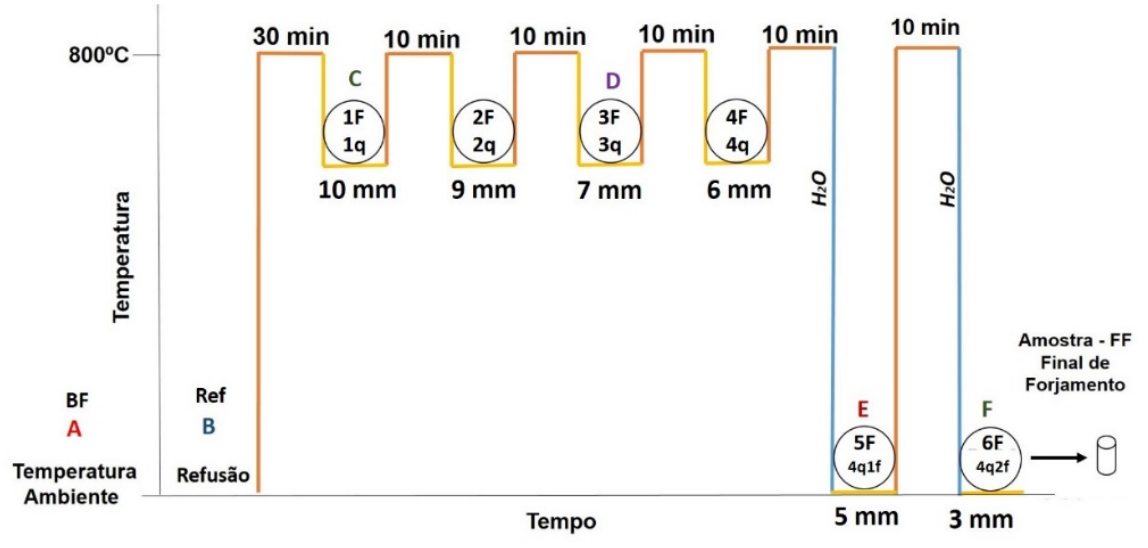

Figura 1. Processamento termomecânico do lingote de uma liga NiTi rica em Ni, com etapas de forjamento rotativo, temperatura/tempos de encharque dos tratamentos térmicos intermediários e diâmetros das amostras.

Como representado na Figura 1, os diâmetros de cada amostra, para cada passe foram:

$-1^{\circ}$ Passe: $10 \mathrm{~mm}$ (barra coletada para análise);

$-2^{\circ}$ Passe: $9 \mathrm{~mm}$;

- $3^{\circ}$ Passe: $7 \mathrm{~mm}$ (barra coletada para análise);

- $4^{\circ}$ Passe: $6 \mathrm{~mm}$; 
- $5^{\circ}$ Passe: $5 \mathrm{~mm}$ (arame coletado para análise);

- $6^{\circ}$ Passe: $3 \mathrm{~mm}$ (arame coletado para análise).

Em resumo, foram selecionadas para estudo neste trabalho as amostras (destacadas por letras na Figura 1):

- Antes do Processamento Termomecânico - Bruta de Fusão (A) e Refusão (B);

- Forjamento a Quente - Primeira (C) e Terceira (D) Etapas de Forjamento;

- Forjamento a Frio - Quinta (E) e Sexta (F) Etapas de Forjamento.

\subsection{Métodos}

\subsubsection{Difração de Raio-X (DRX)}

As amostras para DRX, foram extraídas por meio de um processo de corte abrasivo em uma máquina de corte de precisão com o auxílio de disco diamantado. Após o corte, essas foram submetidas a um lixamento de uma de suas faces, com auxílio de uma lixadeira rotativa manual com lixas de carbeto de silício até 4000 mesh. Posteriormente a etapa de lixamento, as amostras foram imersas em uma solução de $45 \mathrm{ml}$ de $\mathrm{HNO}_{3}, 45 \mathrm{ml}$ de $\mathrm{H}_{2} \mathrm{O}$ e $10 \mathrm{ml} \mathrm{HF}$, por aproximadamente $60 \mathrm{~s}$. Esta decapagem ácida foi realizada com intuito de remover a camada deformada proveniente da etapa de corte e lixamento.

As fases presentes a temperatura ambiente, foram analisadas com o auxílio da análise da difração de raios- $X$. A variação das intensidades dos picos foram medidas com base no acoplamento $\theta / 2 \theta$, variando $2 \theta$ de $30^{\circ}$ a $70^{\circ} \mathrm{com}$ passo de $0,08^{\circ}$ num modo de varredura contínuo. Estes ensaios foram realizados no laboratório de Difração de Raios $X$ do Centro de Investigação de Materiais (CENIMAT), na Faculdade de Ciências e Tecnologia da Universidade Nova de Lisboa, pelo difratômetro Rigaku, com goniômetro (movimento de $\theta$ e amostra 2 $\theta$, detector acoplado), gerador de $3 \mathrm{~kW}$ e ampola selada de $\mathrm{Cu}$, aplicando uma tensão de 40KV e corrente de $30 \mathrm{~mA}$.

\subsubsection{Calorimetria diferencial de varredura (DSC)}

Similar as amostras de DRX, foram extraídas por corte de precisão, com massa entre 10 a $30 \mathrm{mg}$ para análise de DSC. Após esta etapa de corte, as amostras foram submetidas à mesma decapagem ácida descrita na preparação das amostras de DRX.

Para as análises de DSC foi utilizado um equipamento Netzsch modelo F401, Proteus, com forno de baixa temperatura $\left(-150^{\circ} \mathrm{C}\right.$ a $\left.600^{\circ} \mathrm{C}\right)$, instalado no Centro de Investigação de Materiais (CENIMAT), na Faculdade de Ciências e Tecnologia da Universidade Nova de Lisboa. Foram adotados ciclos térmicos entre $-150^{\circ} \mathrm{C}$ e $150^{\circ} \mathrm{C}$ e taxa de aquecimento e resfriamento de $10^{\circ} \mathrm{C} / \mathrm{min}$. As temperaturas de início e fim das transformações de fase foram analisadas a partir dos gráficos gerados pelos ensaios de cada uma das amostras nas distintas etapas do processamento em estudo.

\section{RESULTADOS E DISCUSSÃO}

Pode-se observar na Figura 1 os resultados obtidos a partir da análise de DSC. As temperaturas e sequência de transformação de fase, que foram extraídas das curvas de DSC das diferentes amostras do material em estudo, podem ser numericamente observadas na Tabela 1 em comparação com os gráficos exibidos na Figura 1. 
$\mathrm{Na}$ Tabela 1 são apresentados os valores para início e fim das temperaturas de transformação de fase, indicando as temperaturas da transformação martensítica $\left(\mathrm{Mi}_{\mathrm{i}}\right.$ - Temperatura de Início, $A_{p}$ - Temperatura de Pico Correspondente à $50 \%$ da Transformação, e $A_{f}$ - Temperatura de Fim de Transformação) e da transformação inversa $\left(A_{i}-\right.$ Temperatura Inicial da Transformação Austenítica, $A_{p}$ - Temperatura Correspondente à $50 \%$ da Transformação Austenítica e $A_{f}-$ Temperatura Final de Transformação da Austenítica).
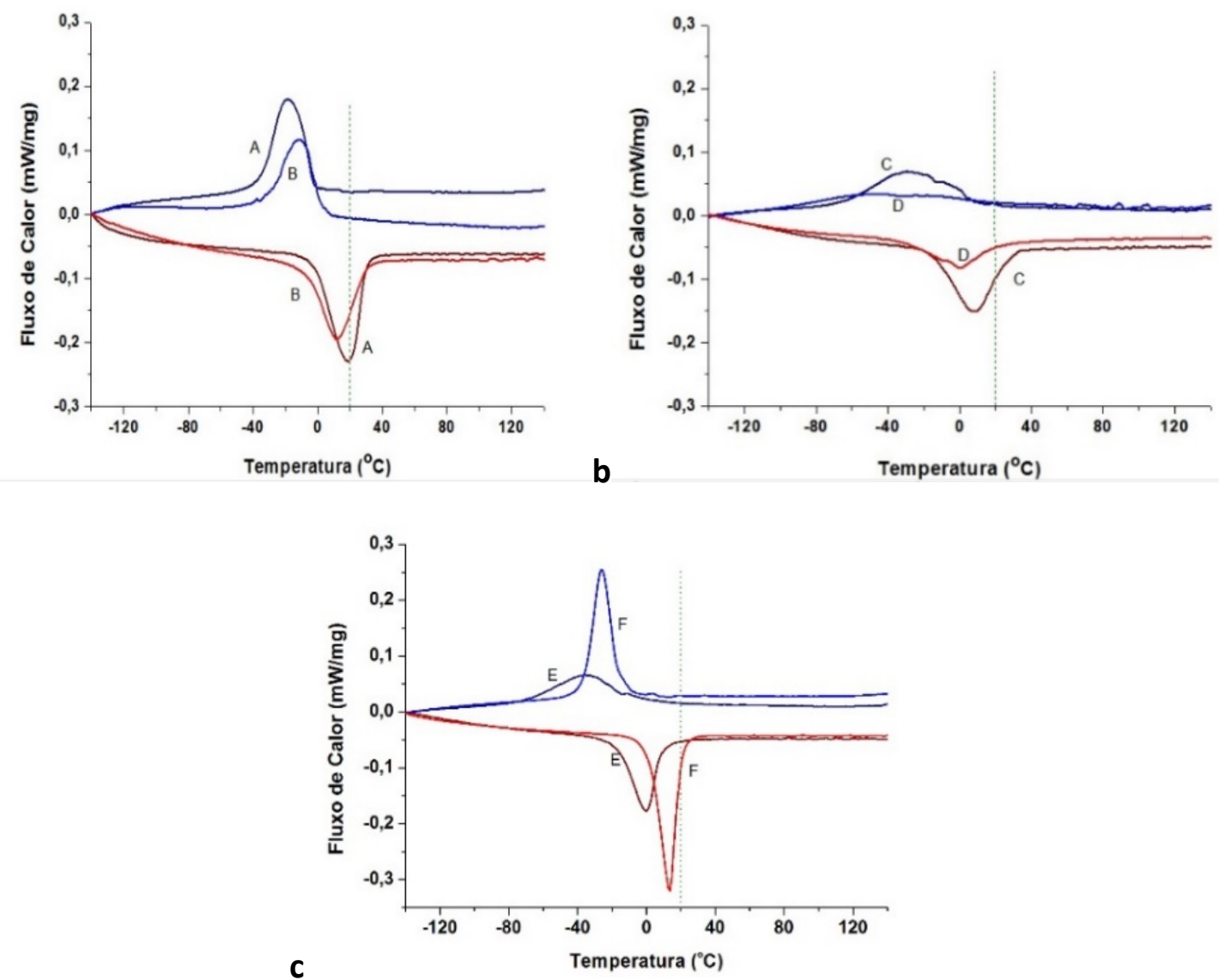

Figura 2. Curva de DSC para liga rica em Ni - a) A - Bruta de Fusão e B - Refusão, b) C - Primeira Etapa (Um Forjamento a Quente) e D - Terceira Etapa (Três Forjamentos à Quente) c) E - Quinta Etapa (Quatro Forjamentos a Quente e Um a Forjamento a Frio) e F - Sexta Etapa (Quatro Forjamentos a Quente e Dois Forjamentos a Frio).

Tabela 2. Valores das temperaturas de Transformação de Fase.

\begin{tabular}{|c|c|c|c|c|c|c|c|c|c|c|}
\hline & \multicolumn{3}{|c|}{ Arrefecimento $\left.\mathbf{(}^{\mathbf{0}} \mathbf{C}\right)$} & \multicolumn{3}{c|}{ Aquecimento $\left(^{\circ} \mathbf{C}\right)$} & \multicolumn{2}{c|}{ Histerese $\left({ }^{\circ} \mathbf{C}\right)$} \\
\cline { 2 - 12 } & $\mathbf{M}_{\mathbf{i}}$ & $\mathbf{M}_{\mathbf{p}}$ & $\mathbf{M}_{\mathbf{f}}$ & Área $(\mathbf{J} / \mathbf{g})$ & $\mathbf{A}_{\mathbf{i}}$ & $\mathbf{A}_{\mathbf{p}}$ & $\mathbf{A}_{\mathbf{f}}$ & Área $(\mathbf{J} / \mathbf{g})$ & $\mathbf{A}_{\mathbf{f}}-\mathbf{M}_{\mathbf{f}}$ & $\mathbf{A}_{\mathbf{i}}-\mathbf{M}_{\mathbf{i}}$ \\
\hline A: BF & $-2,6$ & $-18,3$ & $-34,8$ & 20,71 & $-1,7$ & 18,9 & 29,7 & 21,03 & 64,5 & 0,9 \\
\hline B: Ref & 0,7 & $-10,8$ & $-27,1$ & 16,45 & $-5,8$ & 11,6 & 29,3 & 17,53 & 56,1 & $-6,5$ \\
\hline C: 1F_1q & 8,1 & $-29,4$ & $-63,9$ & 17,88 & $-15,1$ & 9,2 & 27,8 & 16,19 & 91,7 & $-23,2$ \\
\hline D: 3F_3q & - & - & - & - & $-19,2$ & $-3,7$ & 6,9 & 6,75 & - & - \\
\hline E: 5F_4q1f & $-13,4$ & $-35,1$ & $-39,9$ & 11,70 & $-16,5$ & $-0,2$ & 8,4 & 14,62 & 48,3 & $-3,1$ \\
\hline F: 6F_4q2f & $-15,9$ & $-26,0$ & $-36,0$ & 19,73 & 1,7 & 13,4 & 20,2 & 20,3 & 56,2 & 17,6 \\
\hline
\end{tabular}

$$
\text { "-“: Pico indefinido. }
$$

Pelos ensaios realizados pode-se verificar que as temperaturas de transformação de fases das amostras bruta de fusão (A) e refundida (B) apresentam temperatura de final de transformação austenítica acima da temperatura ambiente. Ao relacionar a temperatura de processamento deste material, deformação a quente a partir de 
$800^{\circ} \mathrm{C}$, ou seja, este se encontrava em campos de estabilidade austenítica ao iniciar o processamento termomecânico.

Pode-se verificar nas curvas referentes às amostras $C$ e $D$, que o forjamento a quente referente à primeira etapa de redução, resultou em transformação de fase tanto no resfriamento quanto no aquecimento. No entanto, o terceiro passe de redução (D) resultou numa supressão parcial das transformações, ou seja, durante o resfriamento uma transformação de fase menos perceptível quando comparada com a amostra C. No aquecimento pode-se observar a transformação de fase por um pico bem definido, mas ainda menos evidente quando comparada com a amostra $\mathrm{C}$.

Tendo em vista que ocorre uma redução da temperatura do lingote/barra ao ser retirado do forno, na etapa de reaquecimento para o forjamento rotativo a quente, até o transporte e inserção na forja e que esta redução de temperatura se torna mais expressiva com a redução da seção transversal da barra. Supõe-se que a temperatura de deformação na qual a matriz de B2 foi reduzida progressivamente com progresso das etapas de forjamento rotativo, ou seja, que a temperatura de deformação da amostra $D$ foi menor que a da amostra $C$. Fator este que justificativa a supressão parcial das transformações de fase devido ao encruamento presente na matriz de B2 resultante da terceira etapa de forjamento rotativo a quente.

As amostras correspondentes a pós-condição das etapas de forjamento a frio referem-se ao quinto $(E)$ e sexto forjamento $(F)$, conforme Figura 1c, onde observase a estabilidade da fase B2 à temperatura ambiente. Isso provavelmente se dá pelo teor de níquel existente na liga, suficientemente alto para reduzir a temperatura de transformação martensítica para valores inferiores à temperatura ambiente, a condição adotada para os tratamentos térmicos prévio de solubilização e a quantidade de redução imposta em cada uma das etapas de forjamento rotativo a frio.

Com isso, é suposto que todo o processamento a frio ocorreu no domínio austenítico, pois é sabido que quando se deforma B2 acima do nível de recuperação superelásticas, ou seja, com níveis de deformação plástica associados ao encruamento da estrutura, que ocorrerá um incremento nas temperaturas de transformação martensíticas e de reversão austeníticas ${ }^{(5)}$.

De acordo com os resultados dos ensaios de DRX, apresentados na Figura 3, em confronto com os resultados da análise de DSC (Figura 2), foi possível definir as fases existentes à temperatura ambiente nas amostras em estudo.

Pode-se verificar que as amostras referentes as condições:

- bruta de fusão (A) apresenta picos referentes a fase B2. Ainda é possível observar picos correspondentes a fase B19' (1-11) e (021), no entanto, em função do resultado de DSC que indica que Mi para esta condição é abaixo da temperatura ambiente (Figura 2 e Tabela 1 - BF), a presença dos mesmos deve estar associada a artefato de preparação. Onde a decapagem realizada não foi o suficiente para remover a camada deformada resultante do processo de corte e lixamento durante o preparo da superfície da amostra.

- refusão $(B)$ é possível observar a presença de picos referente a fase B2 ((111), (110), (200) e (211)) e ainda indícios de pico da fase B19' associado ao plano (021). De maneira similar, tendo em vista que o valor de $M_{i}$ para esta amostra é inferior a temperatura ambiente, a presença de B19' deve estar associada a artefato de preparação da superfície da amostra devido a transformação de B2 em B19' induzida pela deformação das etapas de corte e lixamento.

- extraídas após as etapas de forjamento a quente, Primeira Etapa (C - 1F_1q) e Terceira Etapa ( $\left.D-3 F \_3 q\right)$, apresentam fase B2 pelas evidências de picos de 
difração desta fase apenas. No entanto, os picos (200) e (211) do difratograma para a primeira etapa de processamento são mais intensos e o pico (110) menos alargado do que relativos a terceira etapa (Figura 3b). Além disso, o difratograma da terceira etapa apresentam também evidencias de picos de difração de B19' (1-11) e (021). Neste caso se for observado o aspecto dos picos de resfriamento no DSC para as amostras $C$ e $D$ (Figura $2 b$ ) em comparação a definição da temperatura $\mathrm{Mi}$ (destacada na Tabela 1 - 1F_1q e 3F_3q), existe uma possibilidade do método clássico de definição da temperatura $(5,6, \overline{7}, \overline{8})$ ter se afastado muito da real temperatura de início devido ao formato dos picos. Na realidade a transformação martensítica ocorrer ao redor da temperatura ambiente.

a
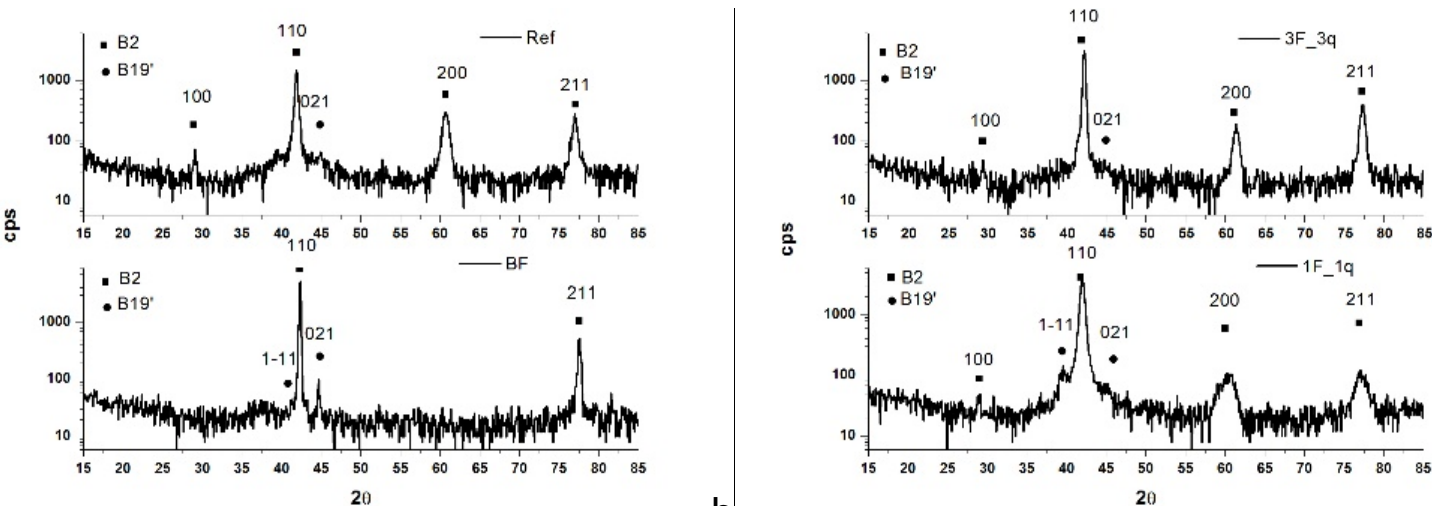

b

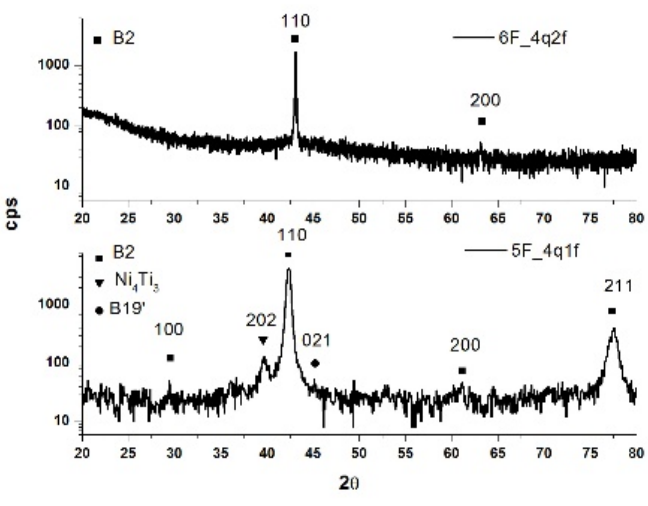

C

Figura 3. Difratograma para liga rica em $\mathrm{Ni}$ - a) $A$ - Bruta de Fusão e $B$ - Refusão, b) $C$ - Primeira Etapa (Um Forjamento a Quente) e D - Terceira Etapa (Três Forjamentos à Quente) c) E - Quinta Etapa (Quatro Forjamentos a Quente e Um a Forjamento a Frio) e F - Sexta Etapa (Quatro

Forjamentos a Quente e Dois Forjamentos a Frio).

- extraídas após as etapas de forjamento a frio, Quinta Etapa de Forjamento (E $\left.5 F \_4 q 1 f\right)$ e Sexta Etapa de Forjamento ( $F$ - 6F_4q2f), apresentam de maneira majoritária a fase B2 (110), com alta intensidade. Contudo, a amostra da quinta etapa contém um único pico de difração detectado além de ser referente a fase B2 está associado ao plano (110) da fase, indicando um possível desenvolvimento de textura na mesma. Enquanto, a amostra da sexta etapa apresenta evidencias de picos mais intensos e alargados de B2 associados aos planos (110) e (211) em conjunto com pico (002) da fase B19' (021). De maneira similar as amostras provenientes as etapas após forjamento a quente, os picos no resfriamento para as amostras após forjamento a frio (Figura 2c) apresentam indício de início de incremento do fluxo de calor muito próximo a temperatura ambiente, enquanto o método clássico utilizado para definir as temperaturas de transformação indicou 
valores de $\mathrm{Mi}_{\mathrm{i}}$ muito inferiores a esta. Dessa forma, existe a possibilidade do pico da fase B19' identificado na amostra $E$ (Figura 3c - 5F_4q1f) não ser artefato da preparação. Ainda foi possível observar a presença de precipitado $\mathrm{Ni}_{4} \mathrm{Ti}_{3}$, para a amostra correspondente a quinta etapa de forjamento, esse precipitado é comum neste tipo de liga, devido as condições de processamento aplicado, uma vez que este é imposto à temperaturas e processamentos capazes de promover tal precipitação metaestável ${ }^{(5,6,7,8)}$.

\section{CONCLUSÃO}

Pelos resultados de DSC e seu confronto com os resultados de DRX apresentados, pode-se concluir que o processamento em questão foi realizado em todas as etapas em campo austenítico. A primeira etapa de forjamento foi realizada na temperatura correspondente a proposta ao processamento $\left(800^{\circ} \mathrm{C}\right)$, no entanto pelos resultados, pode-se observar que a redução da temperatura entre as etapas foi nítida. Ao retirar o material do forno, nas etapas de reaquecimento e transportá-lo para o forjamento rotativo a quente, essa fica explícita levando em consideração a redução dimensional do material. As etapas de forjamento a quente posteriores a primeira etapa de forjamento a quente, foram realizadas em temperaturas inferiores à temperatura de correspondente a temperatura do primeiro forjamento a quente, devido ao incremento na perda de calor em função da redução da seção transversal da barra ao longo das etapas de forjamento.

As etapas referentes as etapas de forjamento a frio, foram realizadas em campo de estabilidade austenítica. Observado pelas características das amostras, tais como: teor de níquel, condição de tratamento térmico prévio de solubilização e temperaturas de transformação martensítica após o processamento ser inferior a temperatura ambiente em função do trabalho mecânico imposto. Estas etapas de forjamento a frio ainda foram capazes de promover um encruamento na matriz B2, devido a grau de redução que resultou em deformação permanente.

\section{Agradecimentos}

A.S.P, S.B.R e P.F.R agradecem a bolsa financiada pela CAPES (APQ-1 2009/02 E26/110.414/2010, APQ-1 2011-2 E-26/110.269.2012, E-26/111.435/2012 - CsF/ Brasil - IBEX 11943-13-0)

P.F.R e F.M.B.F agradecem o financiamento do CENIMAT/I3N pelo COMPETE 2020, através da FCT, pelo projeto UID/CTM/50025/2013. Os autores agradecem o Professor J. Otubo e sua equipe, pela doação do material de partida e pelo apoio e uso da infraestrutura do Instituto Tecnologia da Aeronáutica para realização da fusão e o processamento de forjamento realizado neste trabalho.

\section{REFERÊNCIAS}

1 K. Otsuka, X. Ren, Physical metallurgy of Ti-Ni-based shape memory alloys, Prog. Mater. Sci. 2005, 516, 669.

2 J. Otubo, O.D. Rigo, A.A. Coelho, C.M. Neto, P.R. Mei, The influence of carbon and oxygen content on the martensitic transformation temperatures and enthalpies of $\mathrm{NiTi}$ shape memory alloy, Mater. Sci. Eng. A 2008, 481-482, 639.

3 G.E. Dieter, Mechanical Metallurgy, New York: McGraw-Hill,1986. 
4 J. Otubo, O.D. Rigo, C.M. Neto, P.R. Mei, The effects of vacuum induction melting and electron beam melting techniques on the purity of NiTi shape memory alloys, Mater. Sci. Eng. A 2006, 679, 2006.

5 A.S. Paula, Tratamentos termomecânicos de ligas do sistema Ni-Ti. Doctoral Thesis. UNL/FCT. Lisbon, 2006

6 S.A. Thompson, An overview of nickel-titanium alloys used in dentistry. International Endodontic Journal, 2000, 33, 297-310.

7 D.E. Hodson, W.U. Brown, W. Jefrey, Using Nitinol Alloys. Shape Memory Applications, Inc. 2000.

8 C. Libenson, et al. Differences in the pseudoelasticity behaviour of NiTi orthodontic wires. Journal of Materials Science: Materials in Medicine,1993, 281-84. 